\title{
LIX. On an electrochemical method of investigating the field of electrolytic action
}

\section{Alfred Tribe}

To cite this article: Alfred Tribe (1881) LIX. On an electrochemical method of investigating the field of electrolytic action, Philosophical Magazine Series 5, 11:70, 446-451, DOI:

10.1080/14786448108627041

To link to this article: http://dx.doi.org/10.1080/14786448108627041

册 Published online: 28 Apr 2009.

Submit your article to this journal ¿

Џ Article views: 2

Q View related articles $\sqsubset$ 
Another wife of a son-in-law of a person cannot be the daughter of the person.

A step-child of a sister of a woman cannot be the child of the woman.

A child of a sister of a step-mother of a person cannot be the person.

A sister of a wife of a husband of a woman cannot be the woman.

A parent of another wife of a son-in-law of a person cannot be the person.

A wife of a husband of a sister of a woman cannot be the woman.

A child of a husband of a sister of the mother of a person cannot be the person.

LIX. On an Electrochemical Method of Investigating the Field of Electrolytic Action. By ÁLFED TRIBE* *

$\mathrm{T}$ HE electrochemical method of investigating the field of electrolytic action has for its basis new facts, the nature of which I propose to set forth in the first part of this communication, reserving the second part for the description and discussion of the results which have recently accrued from its application.

\section{The Method.}

When a rectangular plate of metal unconnected with the battery is placed lengthwise in an electrolyte undergoing electrolysis, the plate does work identical in kind with that being done by both electrodes. The electropositive ion of the electrolyte separates and distributes itself on a portion of the plate nearer the + electrode, and the electronegative ion on another part of the plate nearer the - electrode. The respective boundaries of these ions are sharply defined, and the intermedial space free from either ion.

When an aqueous solution of copper sulphate is electrolyzed with silver electrodes, copper of course separates on the - electrode; but more or less of the dark-grey or black silver peroxide forms on the + electrode. $\frac{1}{100}$ of a weber in one minute produces, in fact, a sensible separation of copper and a sensible formation of silyer peroxide on a silver plate 34 millim. $\times 7$ millim.

It follows, therefore, that a silver plate placed in a solution of copper sulphate, under the conditions named in the first paragraph, should have copper deposited on that part which may be supposed to receive - electrification, and silver peroxide on that which receives the positive. Such is the case.

The registration of any set of electrifications in this way by the ions of electrolytes need take only a few minutes, the minimum time being determined by the dimensions of the analyzer $\dagger$, strength of electrolyte, and available current.

* Communicated by the Author, having been read at the Meeting of the French Association, Algiers, April 1881.

$\dagger$ The rectangular silver plate is named, for convenience, the analyzing plate, or, in brief, the analyzer. 
After the electric energy has done enough work on the analyzer for the action to be visible, time is seen to exert no influence in determining the magnitudes of the distributions. Thus in 6 minutes and 60 minutes respectively, identical distributions were recorded by similar analyzers, other circumstances being the same. But the magnitudes of the distributions registered by an analyzer of given dimensions vary with every condition which may be supposed to alter the chemical or physical state of the electrolytic medium, as temperature, proportion of water, and electric quantity.

When at ordinary temperatures the analyzer is placed with its length perpendicular to the electrodes in a homogeneous field*, although the superficial magnitudes of the ions vary, as stated, with any variation in the condition of the electrolytic medium, yet in every case one quality is seen to obtain, namely a similarity of the same ion on the two sides of the analyzer, both as regards magnitude and configuration of its boundary-line. This happens whether the plate has its shorter edge vertical or is supported with its sides horizontal. Under these circumstances the boundaries of the ions are practically parallel with the shorter edge. Distributions having all these characteristics are named parallel (figs. 1 and 3 ).

When the course of the energy makes an oblique angle with the edges of the analyzer, but remains parallel to the sides of the latter, the electrifications recorded are also the same on the two sides of the plate; but the boundary-lines of the ions now cross the plate obliquely to its shorter edge (figs. 4,5 , and 6 ). The positive ion on the longer edge in opposition to the course of the energy is greatest in length, while the negative ion on the same edge of the plate is smallest in length. The obliquity and the intermedial space between the ions increase as the longer edge of the analyzer approaches a line at right angles to the direction of the influence. But at whatever angle the plate is fixed, the boundary-lines of the ions are parallel with the electrodes, and therefore at right angles to the direction in which the energy is transmitted.

When the electric power makes an oblique angle with the sides of the analyzer, the electrifications recorded present a totally different character. The magnitudes of the same ion and the configuration of its boundary-line are now very different on the two sides of the plate. On the side in opposition to the direct course of the energy, the configuration of the positive ion is markedly convex and greater in magnitude than it is on the reverse side of the plate, where, more-

* That is, where the electrodes are of the same depth and breadth as the cross section of the electrolyte. 
over, the configuration of this same ion is markedly concave (figs. 2, 2). Further, the configuration of the boundaryline of the negative ion on the first-named side of the analyzer is concave and smaller in magnitude than on its reverse side, where again the boundary-line of the ion is convex. The intermedial space between the ions increases as the analyzer approaches a position at right angles to the right line between the electrodes, and the characteristic boundary configurations become more and more marked. At right angles the positive ion is smaller in quantity, and arranged longitudinally along the centre of the side of the analyzer facing the + electrode, while the negative ion on the reversed side is similarly disposed, but generally not so well defined.

The classes of distribution described in these two last paragraphs are named non-parallel. The dotted lines in the annexed diagram exhibit the boundary-lines and general characteristics of the parallel and non-parallel distributions.

With shorter edge of analyzer vertical.

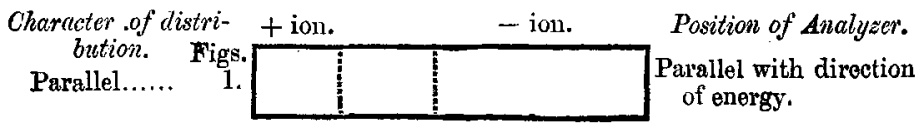

Side in opposition to direction.

Non-parallel.

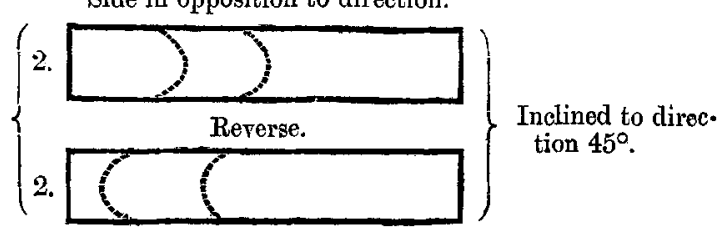

With sides of analyzer horizontal.

Parallel......

3.

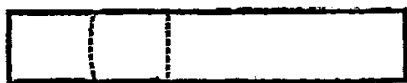

Parallel with direction.

Non-parallel. $(4$

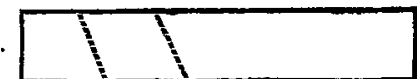

Edge $(t)$ in opposition to direction $15^{\circ}$.

(a)

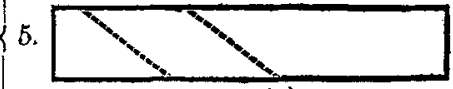

do

$45^{\circ}$.

(a)

6.

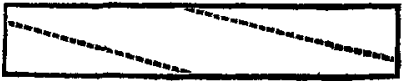

(a) 
It is obvious that the results described furnish the data for determining with ease and precision, first, physical differences in parts of an electrolytic field; and, secondly, the direction in which the energy is being transmitted relatively to either side or edge of the analyzing-plate, and therefore of its direction in the electrolytic medium.

\section{Results.}

I. Demonstration of Differences in corresponding parts of Non-homogeneous Fields.-Few phenomena are more generally known than those of the electric discharge. The feature common to these phenomena is a well-marked differenoe between the spaces in the immediate vicinity of the + and - electrodes. No differences, as far as I am aware, have hitherto been shown to obtain in corresponding parts of electrolytic media. But the supposition that dielectrics and electrolytes differ in degree rather than in kind, as regards the mode in which they transmit the electric influence, is founded on the analogy in the results obtainable in the two media, and is supported by the continuity in electrical qualities evidently prevalent among substances in general. Differences in corresponding parts in the vicinity of the + and - electrodes in certain electrolytic fields might not unreasonably therefore be expected to exist; and the question naturally arose whether the method of research, the principles of which have been set forth, would reveal such differences.

In a homogeneous field, analyzers* placed perpendicular to the electrodes in any part of the field showed that they were surrounded by the same conditions; that is, the magnitades of the ions on an analyzing plate were identical close to the - electrode, close to the + electrode, and midway between the electrodes. But on passing to non-homogeneous fields (i.e. where the electrodes are smaller than the transverse section of the electrolyte) this was no longer the case. The magnitudes of the ions on exactly similar analyzers placed perpendicular, in the centre of, and close to these electrodes, in exactly corresponding positions, were markedly different, and the difference increased as the width of the electrodes relative to the cross section of the electrolyte was reduced.

Differences in corresponding parts of a non-homogeneous field in the vicinity of the electrodes of opposite name are demonstrable, then, by this method of working, and also that the differences are in some way connected with the power of

* In all the experiments to be described, the analyzer was placed with its shorter edge vertical in the electrolytic medium. 
extension of the influence, as no difference whatever is detectable in corresponding parts of a homogeneous field.

II. Demonstration of the Direction in Electrolytic Fields.It follows from the generally received views of electric conduction that, in every part of a homogeneous electrolytic field, the energy is transmitted perpendicularly to the electrodes. The electrochemical method shows, at least, that the direction in this case is everywhere identical. Also it follows that, in certain parts of non-homogeneous fields, the direction is curvilinear. A direct experimental proof of differences in direction in a non-homogeneous field was furnished by the analysis of one of considerable dimensions. In a line joining the centre of the electrodes in this experiment and in another at right angles at its centre, parallel distributions were recorded by analyzers placed lengthwise perpendicular to those sides of the cell where the electrodes were situated. Analyzers placed, however, in the same direction, but a few millimetres from the sides of the cell, and at various distances from the electrodes, recorded non-parallel distributions. It is demonstrable, then, that, in the positions just named, the direction of transmission of the influence forms an angle more or less acute with the sides of the analyzer. Whether the direction results from emission of the influence at various angles from the plane surface of the electrodes, or leaves them always at right angles, and is subsequently driven out of its direct course by the resistance of the medium, remain to be determined.

It may be asked whether the facts just set forth could have been discovered by any other method than the one now described. No other method of which I am aware is capable of demonstrating so directly the direction of the influence in the several parts of a non-homogeneous field. Whether the method employed by De La Rive in 1825, or the one used a few years ago by Prof. Adams, for investigating the laws of electric distribution in electrolytes, is capable of exhibiting all physical differences in the corresponding parts of non-homogeneous fields cannot even be conjectured, until the relation between the magnitudes and the quantities of the ions on the analyzer in these parts of the field has been studied.

Whatever applications of this graphic method may be found, it possesses the advantages of great simplicity, and of the results being self-recorded and permanent. It may be expected from its nature to assist in the demonstration and elucidation of the laws of transmission of electricity through electrolytic media, and in revealing, it is hoped, the inner nature of the electrolytic process itself. 
$A$ detailed account of the results obtained by this method, including determinations of the electric distribution on metallic conductors, will be found in a paper recently communicated by me to the Royal Society.

Dulwich College, April 1881.

LX. On the Law of Force between Electric urrents. By H. W. Watson and S. H. BurburY*.

1. TTHE laws of mutual action between electric currents, or

1 between separate elements of electric currents, have been investigated by Ampère, and, following him, by F. E. Neumann, Weber, Helmholtz, Clausius, and others. Their object has been to discover a law of force between the elements which should give results in accordance with facts established, or supposed to have been established, by experiment. Ampere's experimental data are given by Professor Tait in his work on Quaternions, second edition, p. 250, as follows, using his own words :-

"I. Equal and opposite currents in the same conductor produce equal and opposite effects on other conductors; whence it follows that an element of one current has no effect on an element of another which lies in the plane bisecting the former at right angles.

"II. The effect of a conductor bent or twisted in any manner is equivalent to that of a straight one, provided that the two are traversed by equal currents, and the former nearly coincides with the latter.

"III. No closed circuit can set in motion an element of a circular conductor about an axis through the centre of the circle and perpendicular to its plane.

"IV. In similar systems traversed by equal currents the forces are equal."

To these canons, deduced from experiments of Ampère, may now be added:-

V. Oersted's experiments, showing that the action of a closed circuit is equivalent to that of a magnetic shell of proper strength whose boundary coincides with that of the circuit.

Also VI., an experiment of which the details are given in Maxwell's 'Electricity,' vol. ii. p. 149, which is supposed to prove directly that the force exerted by any closed electric circuit on any element of another circuit is always normal to the element. The law of force, whatever it be, must satisfy these conditions.

* Communicated by the Authors. 